\title{
http://revistainvestigacionacademicasinfrontera.com
}

\section{COMPETENCIAS LABORALES PARA LA INNOVACIÓN EN TRABAJADORES DEL SECTOR SALUD: UN ESTUDIO EXPLORATORIO}

\author{
Jorge Guadalupe Mendoza León \\ Universidad Autónoma de Baja California-Instituto Tecnológico de Sonora, México. \\ jorge.mendoza@itson.edu.mx \\ CARLOS RAÚL NAVARRO GONZÁLEZ \\ Universidad Autónoma de Baja California, Facultad de Ingeniería, Mexicali, B.C., México. \\ cnavarro51@uabc.edu.mx \\ ISRAEL SAUCEDA MEZA \\ Universidad Autónoma de Baja California, Facultad de Ingeniería, Mexicali, B.C., México. \\ isauceda@uabc.edu.mx
}

\section{Resumen}

El Estudio se orienta a explorar Competencias Laborales para la Innovación que poseen trabajadores en hospitales del sector salud. Se definió un instrumento diagnóstico de identificación de Competencias de Innovación, determinando tres dimensiones a analizar: Conocimiento, Habilidades, Actitudes - Valores. Los resultados obtenidos identifica una correlación altamente significativa entre las variables estudiadas, además de determinar 4 niveles de competencias poseídas que conllevan a concluir que aún cuando se percibe una correlación positiva entre las dimensiones de las competencias laborales no hay referencia de un modelo estructurado que guíe sistemáticamente la gestión del proceso de innovación.

This Study is oriented to explore Labor Competency for the Innovation that business have in the health sector. A Labor skills diagnostic identification instrument was defined, determining three dimensions to analyze: Knowledge, Skills and Attitude - Values. The results obtained identify a 


\section{http://revistainvestigacionacademicasinfrontera.com}

highly significant correlation between the studied variables, also determine 4 owned skills levels that lead to conclude that, even when it can be perceived a positive correlation between the labor skills dimension, there's no reference of a structured model that systematically guides the consolidation of grant services process or improve the ones existent.

Palabras clave: Competencias - Innovación - Competencias Laborales - Sector Salud.

\section{Introducción}

En décadas recientes la innovación se ha convertido en factor determinante para la competitividad de las empresas, y en ocasiones, para la subsistencia de las mismas. Es así que la mayoría de las investigaciones en este tema están focalizadas hacia las grandes compañías o empresas que realizan actividades formales de investigación (Escorsa y Valls, 2005) y (Benavides y Quintana, 2002), representando un área de oportunidad abordar este tipo de estudio para la micro, pequeña y mediana empresa. Las capacidades organizacionales y tecnológicas juegan un rol importante en los procesos de innovación, al involucrar el conjunto de habilidades con que cuenta una empresa para usar de manera eficiente el conocimiento técnico adquirido, para asimilar, utilizar y adaptar tecnologías existentes y la creación de nuevas tecnologías para el desarrollo de nuevos productos y procesos (Lall, 1992); (Dutrénit y Vera-Cruz, 2002).

El origen del enfoque de las competencias se vincula con tres procesos sociales significativos: la sociedad del conocimiento, el movimiento de la calidad de la educación y la formación del capital humano. El enfoque se orienta hacia la adquisición de conocimiento y con base en ello, el desarrollo de habilidades fundamentadas en actitudes y valores. Así, las capacidades que se poseen son a su vez competencias (Tobón y Tobón, 2006).

A este respecto (Martens, 2015) en un artículo publicado por la Organización de Estados Iberoamericanos (OEI) señala que las trayectorias de innovación son la base para la mejora sostenida 


\section{http://revistainvestigacionacademicasinfrontera.com}

de la productividad en las organizaciones, además de reconocer que las empresas no siguen un patrón o modelo único, ni son aplicadas por igual, siendo esto parte de la naturaleza de cada empresa y de cada caso en particular. Aun así, es posible caracterizar algunas trayectorias que permitan identificar y entender la evolución de la competencia laboral en las organizaciones. Es esto precisamente, a lo que pretende contribuir el presente trabajo de investigación.

Si bien, es importante identificar las trayectorias de innovación, las cuales para este propósito de estudio entendemos como los diferentes aspectos que caracterizan la aplicación de nuevos conocimientos en el contexto de los sistemas productivos, resulta básico el identificar en forma de diagnóstico cuales son las competencias laborales que orientan hacia el desarrollo de dichas trayectorias. Esta situación apunta a que la competencia laboral implica la innovación de otros subsistemas en la gestión del recurso humano.

\subsection{Antecedentes}

La desaceleración económica para América Latina, iniciada en 2010, así como la disminución de los temores sobre alguna crisis en la región, surgidos en la segunda mitad del 2013 e inicios de 2014, van quedando de lado; a pesar de ello, las expectativas para el 2015 (esperado 2.0\% al 2.5\%), no son las mejores, ya que el crecimiento del PIB del 2014 es modesto, ubicándose entre los rangos del $1.0 \%$ y el 1.5\%; considerando que en el 2013 fue del $2.5 \%$ y en 2012 de $2.9 \%$. (OCDE, Banco de Desarrollo de América Latina y CEPAL, 2014).

El crecimiento en la economía para toda América Latina pudiera ser menor, considerando que se está presentando una fuerte desaceleración en China, asimismo, pero con menor medida, por un endurecimiento mayor, en las condiciones financieras impuestas por Estados Unidos. Por lo que la bonanza que había beneficiado de manera recurrente a los países latinoamericanos, por la abundancia de recursos en materia prima y capitales a corto plazo, han creado perspectivas volátiles de la 


\section{http://revistainvestigacionacademicasinfrontera.com}

actividad económica en dicha región, sin poder aterrizarse en un aumento real en tendencias de crecimiento para dichos países. (OCDE, Banco de Desarrollo de América Latina y CEPAL, 2014). En este mismo informe presentado por la OCDE en 2014, se expone que "la educación, las competencias y la innovación son ámbitos clave para que más países de América Latina superen la trampa del ingreso medio y fortalezcan la emergente clase media de la región". Por su parte, la coordinación nacional de servicios profesionales docentes en México, tradujo un informe ejecutivo preparado por la Organización para la Cooperación y el Desarrollo Económico (OCDE), y traducido con fondos de la Agencia de los Estados Unidos para el Desarrollo Internacional (USAID), en donde se proponen que las sociedades actuales, y más aquellas que se encuentran en países subdesarrollados, requieren desarrollar competencias claves, entendiéndose por estas, más que solo conocimientos y destrezas, se requiere de la habilidad para poder enfrentar las demandas complejas, apoyándose en recursos psicosociales (destrezas y actitudes) en contextos particulares. (OCDE y USAID, 2015).

Ya desde 1995, la Comisión Europea, planteaba en su publicación "Libro verde de la innovación" la importancia de este último concepto, como determinante para las empresas, la sociedad y sus gobiernos (acción pública), en él, se menciona que las funciones de innovación son múltiples, los cuales deben estar encaminados para motivar a las empresas hacia objetivos ambiciosos, de largo plazo y conduciéndolos hacia la renovación de las industrias y a la creación de nuevos sectores de actividad económica; llevando a conceptualizar este término en: renovación y ampliación productos y servicios, métodos de producción abastecimiento y distribución, así como, cambios en la gestión, organización, condiciones y cualificación de los trabajadores. (Europea, 2015).

\subsection{Planteamiento del problema}

Dado que la innovación requiere de un proceso de aprendizaje organizacional, donde intervienen factores como la cultura laboral, la influencia del entorno, la educación y formación; resulta relevante 


\section{http://revistainvestigacionacademicasinfrontera.com}

explorar cual es la condición que prevalece en un ambiente donde se atiende una necesidad básica y prioritaria del ser humano: la atención a la salud. Así, surgen dos preguntas a responder:

a) ¿Cuál es la correlación entre los elementos que componen a las competencias laborales para la innovación?

b) ¿Cuál es el nivel de competencias laborales que poseen los trabajadores del sector salud, en Navojoa?

\subsection{Objetivo}

Determinar la relación existente entre los componentes de las competencias laborales para la innovación que poseen los trabajadores del sector salud en Navojoa, mediante la aplicación de una encuesta con base en el instrumento de la STPS, para caracterizar el nivel de competencias que poseen.

\section{FUNDAMENTOS TEÓRICOS}

Cuando se habla de competencias, se genera un abanico de opciones que tratan de describir el término desde varios contextos. El Centro de Investigación para el Desarrollo, A.C. (por sus siglas, CIDAC), realizó una encuesta de competencias profesionales en el año 2014 (Centro de Investigación para el Desarrollo, 2014), en donde contempla el constructo competencia, desde 
Año 10.

Núm. 26

Revista de Investigación Académica sin Frontera

ISSN: 2007-8870

\section{http://revistainvestigacionacademicasinfrontera.com}

distintas aristas, las cuales van desde competencias en cultura general, liderazgo, tecnologías de la información, operaciones y logística, trabajo en equipo, innovación/emprendimiento, entre otras.

Por su parte (Vargas, 2004), retoma algunas definiciones sobre competencia laboral, entre las que se menciona a CONOCER (México), quien la puntualiza como la capacidad productiva de un individuo que se define y mide en términos de desempeño en un determinado contexto laboral, siendo este no solo de conocimientos, habilidades, destrezas y actitudes; son necesarias pero no suficientes para el desempeño efectivo. Por su parte INEM (España), las define como el ejercicio eficaz de las capacidades que permiten el desempeño de una ocupación, respecto a los niveles requeridos en el empleo. El Polform/OIT, las define como la construcción social de aprendizajes significativos y útiles para el desempeño productivo en una situación real de trabajo, que se obtiene no sólo a través de la instrucción, sino mediante el aprendizaje por experiencia en situaciones concretas de trabajo.

Asimismo, en el Libro Verde de la Innovación (EUROPEA, 1995), se plantean una serie de conceptos relacionados con la variable Innovación, entre los que se mencionan: la renovación y ampliación de la gama de productos y servicios, así como de los mercados correspondientes; además, se expresa como la renovación de los métodos de producción, abastecimiento y distribución; por último, se interpreta como los cambios en gestión, en la organización del trabajo, en las condiciones del trabajo y las cualificaciones de los trabajadores.

Por su parte, la Secretaría del Trabajo y Previsión Social, imprime y difunde el Catálogo de Competencias Clave para la Innovación en el Trabajo, Dicho instrumento identifica tres elementos a evaluar: los conocimientos, habilidades, actitudes y valores que el trabajador mexicano debe poseer y que los empresarios y empleadores deben fomentar para promover entornos favorables, a fin de desarrollar la capacidad creativa e innovadora, que contribuya a lograr un desempeño superior en el plano individual, grupal y organizacional (STPS, 2013). El instrumento señala que las competencias están ligadas al cumplimiento de las tres dimensiones: Conocimientos, Habilidades y Actitudes-Valores; entendiéndose por Conocimientos, al conjunto de saberes cronológicos sobre un tema en particular, materia o disciplina. Asimismo, se entiende 


\section{http://revistainvestigacionacademicasinfrontera.com}

por Habilidad a las destrezas y precisiones requeridas para realizar las tareas inmersas en un trabajo, en relación con el nivel de exactitud requerido. Los Valores son definidos como el conjunto de cualidades compartidas por el personal de una empresa, que especifican el comportamiento ideal, y que son la base de la cultura esperada para el cumplimiento de la visión y misión. Por último, las Actitudes, referidas al actuar de un individuo, al dar respuesta de una manera peculiar, a alguna situación presentada en su desempeño profesional.

En este mismo documento, se profundiza en cada elemento, para denotar lo esperado en ellos. Para el caso de Conocimiento, se espera que el personal sea capaz del: aseguramiento de la calidad, cuente con conocimientos técnicos específicos de la función que se realiza, que conozca de estadística básica y de herramientas de medición, que cuente con la capacidad de gestionar recursos y conocimientos, que tenga liderazgo, así como que sepa utilizar las tecnologías de información. En el caso de Habilidades, se espera que tenga la capacidad de: analizar y solucionar problemas, capacidad de adaptación y de aprendizaje, se sepa comunicarse, realice cálculos numéricos y lógicos, que cuente con liderazgo colaborativo, sea negociador, que su trabajo esté orientado al cliente, que cuente con la capacidad de pensamiento conceptual, creativo, crítico y estratégico, que sepa tomar decisiones y trabajar en equipo.

Para las Actitudes y Valores, se espera que el individuo tenga: Autonomía, sea colaborativo y cooperador, tenga compromiso por la calidad y la mejora continua, sea ético, cuente con fortalezas, se identifique e integre con la organización, se deje motivar, sea proactivo, respetuoso por la diversidad y sea incluyente, y que cuente con un sentido de responsabilidad social.

Para los fines de este estudio, se entiende por competencia a la combinación de conocimientos, habilidades, actitudes y valores aplicados a un contexto laboral (STPS, 2013). Estas competencias potencialmente se convierten en capacidades para desarrollar procesos de innovación ya sea en productos, procesos, formas de comercialización, o formas de organizar la producción y administración (OCDE, 2005) y De acuerdo con la STPS (2013), Tobón (2006) y Martens (2015), cada trabajador tiene competencias específicas de acuerdo con su puesto de trabajo y capacidades personales. En consecuencia, el trabajador no necesariamente posee todas las competencias aquí 
Año 10. Académica sin Frontera

Núm. 26

ISSN: 2007-8870

\section{http://revistainvestigacionacademicasinfrontera.com}

mencionadas al máximo nivel. Dicho esto, el enfoque debe ser individual, dado que cada persona tiene habilidades y potencialidades diferentes. 


\section{Julio- Diciembre 2017}

Año 10.

Núm. 26

Revista de Investigación Académica sin Frontera ISSN: 2007-8870

\section{http://revistainvestigacionacademicasinfrontera.com}

\section{METODOLOGÍA}

\subsection{Participantes}

La investigación es de tipo transversal exploratoria y descriptiva de corte cuantitativo. Los participantes son trabajadores de hospitales y clínicas médicas de la región sur de Sonora, de manera que se ha adaptado un instrumento con tres dimensiones a saber: conocimiento, habilidades y actitudes-valores. Y dentro de dichos factores se encuentran las competencias de Metodologías de calidad; Conocimiento técnico de la función; cálculos numéricos y herramientas de medición; Gestión de recursos; Gestión del conocimiento; Liderazgo; tecnologías de información y comunicación; Análisis y solución de problemas; Capacidad de adaptación; Capacidad de aprendizaje; Comunicación; Orientación al cliente; Pensamiento creativo; Toma de decisiones; Trabajo en equipo; Negociación y fortaleza; Identificación e integración con la organización; Motivación; Respeto a la diversidad y participación incluyente y la Responsabilidad social.

Se asume un muestreo no probabilístico por conveniencia, debido a que el presente trabajo es un estudio piloto el cual pretende observar el comportamiento de la escala utilizada y adaptada para fines del presente. Por lo que se validará en tres de los cinco hospitales ubicados en el sur de Sonora. Cada institución supone una muestra de 15 personas de diferentes áreas y funciones (médicos, enfermeras, administrativos, técnicos e intendentes), con el propósito de recabar la información. La escala tiene aproximadamente una duración de entre 10 y 15 minutos máximos y se concreta a explorar aspectos técnicos acerca de los conocimientos y habilidades que se poseen en relación a sus competencias de innovación.

\subsection{Materiales}




\section{http://revistainvestigacionacademicasinfrontera.com}

El Instrumento, consta de 80 ítems, divididos en 3 dimensiones, y 7, 9 y 4 subdivisiones, respecto a cada una de las dimensiones respectivamente, cada una con 4 reactivos, que miden el nivel de dominio, los cuales se identifican de la siguiente manera: Nivel D (aplica competencias a un reducido grupo de tareas sencilla, en procesos normalizados, rutinarios y predecibles, requiriendo a veces supervisión en el trabajo); Nivel C (competencias en grupos variados de tareas, y en distintos contextos con total autonomía); Nivel B (aplica competencias con un alto grado de responsabilidad y supervisando el trabajo de otros en diversos contextos y tareas), y Nivel A (Competencia en un amplio conjunto de actividades complejas, con responsabilidad de supervisar trabajo en equipos). Cada una de estas opciones de respuesta cuenta con una escala de tipo Likert, que va desde Siempre, en la primera opción hasta Nunca, pasando por Casi siempre y Casi nunca.

Las dimensiones que se manejan en este instrumento sobre competencia son tres: 1) Conocimiento, con 7 sub dimensiones que son: (Metodologías de calidad, Conocimientos técnicos específicos de la función, Cálculos numéricos, estadística y herramientas de medición, Gestión de recursos, Gestión del conocimiento, Liderazgo y, Tecnologías de la información y comunicación); 2) Habilidades, con 9 sub divisiones, que son: (Análisis y solución de problemas, Capacidad de adaptación, Capacidad de aprender, Comunicación, Orientación al cliente, Pensamiento creativo, Toma de decisiones, Trabajo en equipo, Negociación y fortaleza).

3) Actitudes y valores, con 4 sub divisiones, que son (Identificación e integración con la organización, Motivación, Respeto a la diversidad y participación incluyente, Responsabilidad social). Inicialmente las 3 dimensiones se construyen con un total de 78 items, clasificados en las tres dimensiones, mismos que con base en el análisis de reactivos y tamizando el instrumento se eliminaron aquellos que no representaban información relevante para el contexto y sujeto de estudio, quedando un total de 57 items como base para el análisis. 


\section{http://revistainvestigacionacademicasinfrontera.com}

\subsection{Procedimiento}

Se aplicaron 45 cuestionarios in situ en 3 hospitales de Navojoa, entre la muestra recolectada se consideraron los distintos puestos y funciones, independientemente del grado y nivel de formación académica; conformando una base de datos elaborada y procesada con el software SPSS, versión 19. El presente instrumento fue analizado en su aplicación por medio de validez concurrente, la cual fue obtenida por medio de la técnica de grupos contrastados, a través de la prueba Chi Cuadrada, con valores cuya significancia asintótica bilateral fue menor a 0.05, en donde los 57 reactivos cumplieron con el requisito de la distribución de frecuencias encontradas; es decir, las frecuencias más altas y bajas estuvieron en los extremos (superior para el grupo alto e inferior para el grupo bajo). Asimismo, con una prueba t de Student se corrobora que los 57 reactivos tienen $\mathrm{p}$ asociados menores al 0.05 . Siguiendo la lógica del análisis factorial para establecer la validez de constructo, se efectuó un análisis con Varimax con káiser, identificándose 3 dimensiones, a saber: Conocimiento, Habilidades, Actitudes y Valores. Posteriormente, se identificó la confiabilidad del instrumento a través de consistencia interna de la escala, la cual mostró un valor de $\mathrm{r}=0.889$, medida por el coeficiente alfa de Cronbach.

Asimismo, se analizó la correlación entre las tres dimensiones de estudio, de manera que la variable Conocimientos presenta una correlación positiva altamente significativa con relación a las habilidades, esto significa que al incrementar la base de conocimientos, se tendrán mayores competencias o habilidades laborales para generar innovaciones. Esto conlleva a revisar el esquema que cada ente estudiado utiliza en sus procesos de aprendizaje y acumulación de conocimiento que permitan la gestión efectiva del mismo y la adquisición de las competencias y el diseño de una dinámica para impulsar la innovación. Esto se aprecia en la introducción de nuevos servicios como es el caso de uno de los hospitales que recientemente incorporó la prestación del servicio de resonancia magnética, siendo el único que lo ofrece en el sur de Sonora (abarcando 3 municipios). 
Núm. 26

ISSN: 2007-8870

\section{http://revistainvestigacionacademicasinfrontera.com}

Para el caso de la relación entre Conocimientos y la variable Actitudes y valores, esta resulta altamente significativa y positiva, lo cual confirma la importancia de manejar la ética y política institucional en el ejercicio diario de los servicios que prestan. Ejemplos que reflejan esta correlación se aprecia en la fortaleza en la característica que define el trato digno, respetuoso y tolerante a las personas, así como el respeto por las normatividad institucional. Las Habilidades y la variable Actitudes y valores presentan una correlación positiva y con alta significancia, al establecer que los trabajadores de los servicios de atención a la salud que prestan los hospitales de la región, son capaces de revisar con sentido crítico las estrategias de la organización en su conjunto y proponer los cambios pertinentes. 


\section{http://revistainvestigacionacademicasinfrontera.com}

Tabla 1 Correlación entre Dimensiones

\begin{tabular}{|c|c|c|c|}
\hline Correlaciones & Conocimientos & Habilidades & $\begin{array}{l}\text { Actitudes } \\
\text { y Valores }\end{array}$ \\
\hline $\begin{array}{l}\text { Conocimientos } \quad \text { Correlación de Pearson } \\
\text { (agrupada) }\end{array}$ & 1 & $.834(* *)$ & $.577(* *)$ \\
\hline Sig. (bilateral) & & .000 & .001 \\
\hline $\begin{array}{l}\text { Habilidades } \quad \text { Correlación de Pearson } \\
\text { (agrupada) }\end{array}$ & $.834(* *)$ & 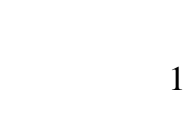 & $.622(* *)$ \\
\hline Sig. (bilateral) & .000 & & \\
\hline $\begin{array}{l}\text { Actitudes y valores Correlación de Pearson } \\
\text { (agrupada) }\end{array}$ & $.577(* *)$ & $.622(* *)$ & 1 \\
\hline Sig. (bilateral) & .001 & .000 & \\
\hline $\mathrm{N}$ & 29 & & 29 \\
\hline
\end{tabular}

** La correlación es significativa al nivel 0,01 (bilateral).

\section{RESULTADOS Y CONCLUSIONES}

El análisis para determinar la relevancia de las variables estudiadas se describe a continuación.

\subsection{Dimensión Conocimiento}

En relación a los datos obtenidos en las dimensiones estudiadas, se encontró que en la variable "Conocimientos" los trabajadores de hospitales de la región sur de Sonora presentan como resultado más alto el "bajo nivel de competencia de innovación” con un 40\%. Esta condición permite asumir que la cultura laboral para promover la innovación no es una de las prioridades a atender en 
Julio- Diciembre 2017

Año 10.

Núm. 26

\section{Revista de Investigación Académica sin Frontera ISSN: 2007-8870}

\section{http://revistainvestigacionacademicasinfrontera.com}

el sector salud y se ve reflejado cuando por ejemplo, en uno de los hospitales se ha realizado una inversión recursos financieros para adquirir tecnología de punta para estudios de resonancia magnética y no se cuenta con el recurso humano que tenga el conocimiento para operarlo. La siguiente frecuencia indica un "alto nivel de competencia de innovación" con un $23.3 \%$, lo cual significa que la mayoría de las distintas actividades que se realizan en los hospitales requieren de un mínimo nivel de formación académica. Luego, se identifica "muy alto nivel de competencia de innovación" con 20\%, el cual de acuerdo con las entrevistas no estructuradas que se obtuvieron durante la aplicación del instrumento, lo caracterizan los perfiles con grado de especialidad en el cuidado de la salud, tanto en áreas administrativas como médicas. Finalmente, un "muy bajo nivel de competencia" con un $13.3 \%$, el cuál es conformado por personal que para su función y puesto no requiere de alto grado de formación (ver tabla 2).

Tabla 2 Resultados Dimensión "Conocimientos"

\begin{tabular}{|l|r|r|r|r|}
\hline Dimensión Conocimientos & Frecuencia & Porcentaje & $\begin{array}{c}\text { Porcentaje } \\
\text { válido }\end{array}$ & $\begin{array}{c}\text { Porcentaje } \\
\text { acumulado }\end{array}$ \\
\hline Muy bajo nivel & 4 & 13.3 & 13.8 & 13.8 \\
Bajo nivel & 12 & 40.0 & 41.4 & 55.2 \\
Alto nivel & 7 & 23.3 & 24.1 & 79.3 \\
Muy alto nivel & 6 & 20.0 & 20.7 & 100.0 \\
Total & 29 & 96.7 & 100.0 & \\
Sistema & 1 & 3.3 & & \\
& 30 & 100.0 & & \\
\hline
\end{tabular}

\subsection{Dimensión Habilidades}

En esta dimensión se observa predominante un "alto nivel de competencia de innovación" con una frecuencia del 40\%, lo cual se entiende como algo lógico dada la formación con alto grado de 
Núm. 26

ISSN: 2007-8870

\section{http://revistainvestigacionacademicasinfrontera.com}

especialización y la experiencia que poseen la mayoría del recurso humano en la entidad estudiada. Esta condición contrasta con la falta de iniciativas para la innovación, ya que se infiere la falta de estrategia para promoverla. El personal categorizado en la frecuencia "Muy bajo nivel de competencia", es sólo el 13\%, hecho que también se intuye en la lógica al tratarse de un número reducido de empleados que no requieren de una formación profesional y especializada para desempeñar sus labores. El alto coeficiente de correlación identificada entre las variables Conocimiento y Habilidades, infiere una estrecha relación entre una base de conocimiento sólida una experiencia desarrollada en la aplicación de los mismos, lo cual desde la perspectiva de los Sistemas de Innovación (Lundvall, 2002) es un factor relevante para innovar (ver tabla 3). 


\section{http://revistainvestigacionacademicasinfrontera.com}

Tabla 3 Resultados Dimensión "Habilidades"

\begin{tabular}{|l|r|r|r|r|}
\hline $\begin{array}{l}\text { Dimensión } \\
\text { Habilidades }\end{array}$ & Frecuencia & Porcentaje & \multicolumn{1}{l}{$\begin{array}{l}\text { Porcentaje } \\
\text { válido }\end{array}$} & $\begin{array}{r}\text { Porcentaje } \\
\text { acumulado }\end{array}$ \\
\hline Muy bajo nivel & 4 & 13.3 & 13.8 & 13.8 \\
Bajo nivel & 8 & 26.7 & 27.6 & 41.4 \\
Alto nivel & 12 & 40.0 & 41.4 & 100.0 \\
Muy alto nivel & 5 & 16.7 & 17.2 & \\
Total & & & & \\
Sistema & 29 & 96.7 & 100.0 & \\
\hline
\end{tabular}

\subsection{Dimensión Actitudes y Valores}

En esta dimensión se observa un "Alto nivel de competencia" (75\%), lo cual indica aspectos como el grado de compromiso y responsabilidad social, motivación al trabajo, identidad e integración con la organización, trabajo en equipo, entre otros, que poseen los trabajadores del sistema hospitalario estudiado. Asimismo, se observa que en algunos casos y aun cuando los trabajadores son fuente de sub contratación, como en el caso de servicios de limpieza y mantenimiento, estos asumen la filosofía de la organización donde desempeñan su labor directa (ver tabla 4). La relación que se lee entre las variables Conocimiento, Habilidades y, las Actitudes y Valores, infiere que para desarrollar una actividad laboral, al menos, el saber qué hacer (conocer) y cómo hacerlo (habilidad) van de la mano con la forma de hacerlo (Actitud). 


\section{http://revistainvestigacionacademicasinfrontera.com}

Tabla 4 Resultados Dimensión "Actitudes y Valores"

\begin{tabular}{|l|r|r|r|r|}
\hline \multicolumn{1}{|c|}{$\begin{array}{r}\text { Dimensión Actitudes y } \\
\text { Valores }\end{array}$} & Frecuencia & Porcentaje & $\begin{array}{c}\text { Porcentaje } \\
\text { válido }\end{array}$ & $\begin{array}{c}\text { Porcentaje } \\
\text { acumulado }\end{array}$ \\
\hline Muy Bajo Nivel & 5 & 16.6 & 16.6 & 16.6 \\
Bajo Nivel & 6 & 20.0 & 20.0 & 36.6 \\
Alto Nivel & 7 & 23.4 & 23.4 & 60.0 \\
Muy Alto Nivel & 11 & 36.7 & 36.7 & 100.0 \\
Total & 29 & 100.0 & 100.0 & \\
& & & & \\
Sistema & 1 & 3.3 & & \\
\hline
\end{tabular}

De acuerdo a los resultados encontrados se puede afirmar que el personal que labora en los hospitales y clínicas médicas, en la región sur de Sonora, presentan una base de conocimientos directamente relacionados con la habilidad para ejercerlo, sin embargo, no existe un modelo de gestión de la innovación formalmente aplicado en las empresas estudiadas, limitando con ello la mejora competitiva, pues no se consolida un proceso formal de innovación, lo cual de acuerdo con Mendoza y Valenzuela (2014) y Medellín (2010) es un factor clave para propiciar innovación de tipo incremental. Los resultados obtenidos significa que en el giro de los servicios de atención a la salud factores como la inversión en equipo e instrumental médico no garantiza que el servicio sea eficiente y eficaz, pues con respaldo empírico se puede afirmar que los trabajadores no tienen la competencia para el uso y aplicación de la tecnología adquirida, esta apreciación se ve respaldad por los estudios de (Jasso y Ortega, 2007), el cual presenta semejanzas con el presente estudio, dando confirmación al hecho de que cuando se habla de competencias para el trabajo, las capacidades tecnológicas son su similar. 
Julio- Diciembre 2017

"El salkes de ni lijas

Año 10.

Núm. 26
Revista de Investigación Académica sin Frontera

ISSN: 2007-8870

\section{http://revistainvestigacionacademicasinfrontera.com}

\section{Conclusión}

El presente estudio permite concluir que aun cuando se perciben una correlación positiva entre las dimensiones de las competencias laborales, no hay referencia de un modelo estructurado que guíe sistemáticamente la consolidación de nuevas formas o procesos de otorgar el servicio o mejorar los ya existentes; por ello, se debe trabajar caracterizar y sistematizar un modelo de gestión de la innovación adecuado al contexto del sector salud. Es precisamente el segundo momento que se pretende abordar una vez que se hayan explorado y definido cuales son las capacidades y potencialidades de uso del conocimiento base que existe y cómo este puede ser aprovechado para la generación de valor tanto al sector hospitalario como a la sociedad a la que le sirve. 
Julio- Diciembre 2017

Año 10.

Revista de Investigación

Núm. 26

Académica sin Frontera

ISSN: 2007-8870

http://revistainvestigacionacademicasinfrontera.com

\section{REFERENCIAS DOCUMENTALES}

CENTRO DE INVESTIGACIÓN PARA EL DESARROLLO, A. 2014. Encuesta de

Competencias Profesionales 2014. Editorial CIDAC. (México)

EUROPEA, C. 1995. Libro Verde de la Innovación. Bruselas: Comisión Europea. Obtenido de Libro Verde de la Innovción.

EUROPEA, C. 2015. Libros verdes - European Commission - Europa. Disponible en http://ec.europa.eu/green-papers/index_es.htm\#1995. Consultado el 05.05.2015

JASSO, J. Y ORTEGA, R. Contaduría y Administración. Acumulación de capacidades tecnológicas locales en un grupo industrial suderúrgico en México. Revista SciELO México. Vol. 223: 69-89.

LUNDVALL, B. 2002. Sistemas Nacionales de Producción Innovación y construcción de Competencias. Editorial Elsevier Science pp 213-231.

MARTENS, L. (2015). Organización de Estados Iberoamericanos. Disponible en http://www.oei.es: http://www.campus-oei.org/oeivirt/fp/01cap02.htm. Consultado el 30.05.2015

MEDELLÍN, E. 2010. Gestión tecnológica en empresas innovadoras mexicanas. Revista de Administración e Innovación. Vol. 7. № 3: 58-78.

MENDOZA, J. y VALENZUELA, A. 2014. Aprendizaje, innovación y gestión tecnológica en la pequeña empresa. Un estudio exploratorio de las industrias metalmecánica y de tecnologías de información en Sonora. Contaduría y Ddministración, FCyA, UNAM. pp 253-284. (México) 
Julio- Diciembre 2017

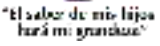

Año 10.

Núm. 26
Revista de Investigación Académica sin Frontera ISSN: 2007-8870

http://revistainvestigacionacademicasinfrontera.com

OCDE. 2005. Manual de Oslo. Editorial Tragsa. Madrid (España)

OCDE y USAID. 2015. Dirección General de Formación y Desarrollo Profesional. Disponible en https://www.google.com.mx/url?sa=t\&rct=j\&q=\&esrc=s\&source=web \&cd=2\&cad=rja\&uact=8 \&ved=0CCIQFjAB\&url=http\%3A\%2F\%2Fformacioncontinua.sep.gob.mx\%2Fsites\%2Fcursoba sico09\%2Fanexos\%2F9Definicion_de_Competencias_Clave.pdf\&ei=_28uVYezHMHEsAXLko CwBw\&usg=AFQjCNHGN. Consultado el 05.05.2015

OCDE, Banco de Desarrollo de América Latina y CEPAL. 2014. Perspectivas económicas para América Latina 2015. Educación, Competencia e Innovación para el desarrollo. (España)

STPS. 2010. Catálogo de competencias clave para la innovación en el trabajo. Gobierno Federal. (México)

STPS. (2013). Instrumento de Identificación de Competencias de Innovación. .Dirección General de Capacitación STPS. (México)

VARGAS, F. 2004. 40 preguntas sobre competencia laboral. Editorial Montevideo: LO/Cienterfor. 